\title{
Experimental evidence of adiabatic splitting of charged particle beams using stable islands of transverse phase space
}

\author{
S. Gilardoni, M. Giovannozzi, M. Martini, E. Métral, P. Scaramuzzi, and R. Steerenberg \\ CERN, AB Department, CH 1211 Geneva, Switzerland
}

A.-S. Müller

FZK-ISS-ANKA, Karlsruhe, Germany

(Received 18 October 2005; published 17 October 2006)

\begin{abstract}
Recently, a novel technique to perform multiturn extraction from a circular particle accelerator was proposed. It is based on beam splitting and trapping, induced by a slow crossing of a nonlinear resonance, inside stable islands of transverse phase space. Experiments at the CERN Proton Synchrotron started in 2002 and evidence of beam splitting was obtained by summer 2004. In this paper, the measurement results achieved with both a low- and a high-intensity, single-bunch proton beam are presented.
\end{abstract}

DOI: 10.1103/PhysRevSTAB.9.104001

PACS numbers: 29.27.Ac, 05.45.-a, 29.27.Bd

\section{INTRODUCTION}

A charged-particles' beam is usually extracted from a circular machine either on a single turn, the so-called fast extraction, or over many million turns, the so-called slow extraction. An intermediate mode is the multiturn extraction, when the beam is ejected over a few turns by shaving on the blade of an electrostatic septum. Recently, a novel method was proposed to perform multiturn extraction [1]: the beam is split by means of stable islands of the transverse phase space, generated by special magnets, sextupoles, and octupoles. The islands are swept through the beam by applying a slow variation of the machine tune. The effect of the tune variation is twofold, as it allows trapping particles inside islands and also controlling their separation. When the beamlets are split, prior to extraction, no particle is left between them and the blade of the extraction septum, thus avoiding any loss due to the particles' interaction with the septum electrodes.

Numerical simulations were performed, showing that the method is efficient in trapping and splitting the beam. Indeed, such an approach can be generalized to arbitrary one-dimensional resonances [2].

The use of stable islands is a key feature as it enables controlling the transverse emittance of the split beam. Furthermore, the process can be time reversed so that a multiturn injection based on stable islands can be envisaged [3]. These features make the proposed approach radically different with respect to slow extraction, where the beam is ejected thanks to the unstable motion near the separatrix of the third-order resonance. In fact, neither can the transverse emittance be chosen, being dictated by the width of the stochastic layer around the separatrix [4], nor can the method be reversed to inject a beam into a circular machine. A confirmation of the theoretical predictions concerning the splitting process would be the first experimental evidence of a new type of beam manipulation, which could be used to control the value of the transverse emittance in a proton synchrotron, in addition to wellknown techniques such as, e.g., stochastic and electron cooling. This novel technique would be the natural generalization of the longitudinal beam splitting [5], a wellestablished technique at the heart of the beam generation for the planned CERN Large Hadron Collider (LHC).

For the sake of completeness, it has to be mentioned that the properties of stable islands were already probed in a series of nonlinear beam dynamics experiments in the late 1980s and early 1990s [6,7]. However, in all the cases the beam was simply displaced into the islands and the transverse emittance was never manipulated as the beam was not split by means of a single-passage resonance crossing, which is the novelty of the study presented in this paper.

The experimental campaign launched at the CERN Proton Synchrotron (PS) in 2002 was successfully completed by the end of 2004 with the evidence of beam splitting by crossing a stable resonance. Out of the large amount of data, the results presented here refer mainly to the 2004 run. Various tests were performed over the threeyear period at the beam momentum of $14 \mathrm{GeV} / c$ [8-10]. The CERN-specific goal consists of applying this technique to reduce drastically the losses intrinsic to the present extraction method for fixed target experiment beams, based on beam slicing onto an electrostatic septum. In particular, this will improve the efficiency of the high-intensity proton beams for the CERN Neutrino to Gran Sasso experiment (CNGS) [11], also in view of a possible intensity upgrade $[12,13]$. These boundary conditions fixed the choice of the resonance used, which is the fourth order so to generate five beamlets, sharing approximately the same intensity ( $20 \%$ of the initial beam).

The plan of the paper is the following: in Sec. II the overall strategy used during the experimental campaign is outlined. In Sec. III the description of the experimental setup is presented, while in Secs. IV and V the results concerning the phase space reconstruction and the beam 
splitting are presented, respectively. The account of the detailed measurements performed to probe the reversibility of the method and the increase of the fraction of trapped particles is reported in Sec. VI. The simple extraction test is reported in Sec. VII. Some conclusions and the discussion of the future studies are presented in Sec. VIII.

\section{MEASUREMENT STRATEGY}

The proposed beam manipulation is, to a large extent, independent on the longitudinal beam structure. Therefore a single-bunch beam was generated for the experimental tests, as this requires less setting-up time and avoids most of the complications related with multibunch operation in the PS machine. The first stage consists in performing the reconstruction of the phase space topology to assess the presence of the stable islands needed to split the beam. This is obtained by means of a pencil beam, i.e., a beam with low intensity and small transverse emittances. The latter property ensures that the beam probes the phase space similarly to a single particle.

After having measured the structure of the transverse phase space, a different type of beam is used to test the splitting process. This second type of beam features low intensity, in order to reduce as much as possible space charge effects that might have an impact on the splitting process (in Ref. [1] the space charge tune spread at nominal intensity was estimated to be of the order of few $10^{-3}$ ). At the same time, the beam has a large horizontal emittance, similar to the one of the nominal beam, i.e., the highintensity required for the CNGS. The need of a large horizontal emittance is twofold: first, it simulates the conditions of the nominal beam, thus easing the task of setting up the resonance crossing for the realistic condition of high intensity, as the machine status will be essentially the same for low and high intensity. Second, a beam with large horizontal emittance has a larger local density far from the center. This implies that the fraction of particles trapped in the islands will be higher, because the islands have large acceptance at large amplitudes. Parenthetically, the generation of the low-intensity, large horizontal emittance beam is rather involved. In fact, it requires the use of a special device between the proton Linac and the PSBooster, the PS injector. Such a device, called sieve, is made by a metallic foil with a series of holes: when it is inserted into the beam passage it reduces the overall intensity and, via scattering, it also induces emittance blowup.

The last part of the experimental measurements consists in repeating the beam splitting tests by using the realistic version of the single bunch with high intensity. As already mentioned, the low- and high-intensity beams used for the splitting tests feature approximately the same value of the horizontal emittance. On the other hand, the vertical one is much larger for the high-intensity beam, thus posing potential issues with nonlinear coupling between the two transverse degrees of freedom. This was the case of the
TABLE I. Main parameters of the various beams used for the experimental studies for the novel multiturn extraction. The value of $\Delta p / p$ refers to $14 \mathrm{GeV} / c$ and $\epsilon_{H, V}(2 \sigma)$ stands for the $2 \sigma$ physical emittance.

\begin{tabular}{lccc}
\hline \hline & $\begin{array}{c}\text { Intensity } \\
\left(10^{10}\right)\end{array}$ & $\begin{array}{c}\epsilon_{H / V}(2 \sigma) \\
(\mu \mathrm{m})\end{array}$ & $\begin{array}{c}\Delta p / p(2 \sigma) \\
\left(10^{-3}\right)\end{array}$ \\
\hline Pencil beam & 40 & $0.46 / 0.42$ & 0.5 \\
Low intensity & 45 & $2.41 / 0.64$ & 0.5 \\
High intensity & 600 & $2.52 / 1.72$ & 1.4 \\
\hline \hline
\end{tabular}

losses observed during resonance crossing with the highintensity beam during the 2003 run (see Ref. [10]). Indeed, it was found that the losses were due to the combined effect of the large vertical emittance together with the maximum value of the vertical beta function reached at the ring location of the octupole used to generate the stable islands. No more losses were observed during the 2004 experimental campaign thanks to the displacement of the octupole to a straight section featuring a minimum value of the vertical beta function.

The beam parameters used in the experimental campaign are reported in Table I.

\section{EXPERIMENTAL SETUP}

As far as the experimental setup is concerned, two sextupoles and one octupole are used to generate the stable islands. They are located in the same straight section (SS) 21 (see Fig. 1 for a sketch of the experimental setup), which is the most suitable among the 100 SS composing the PS lattice. The machine tune is controlled by means of two families of focusing and defocusing quadrupoles almost evenly distributed along the PS circumference.

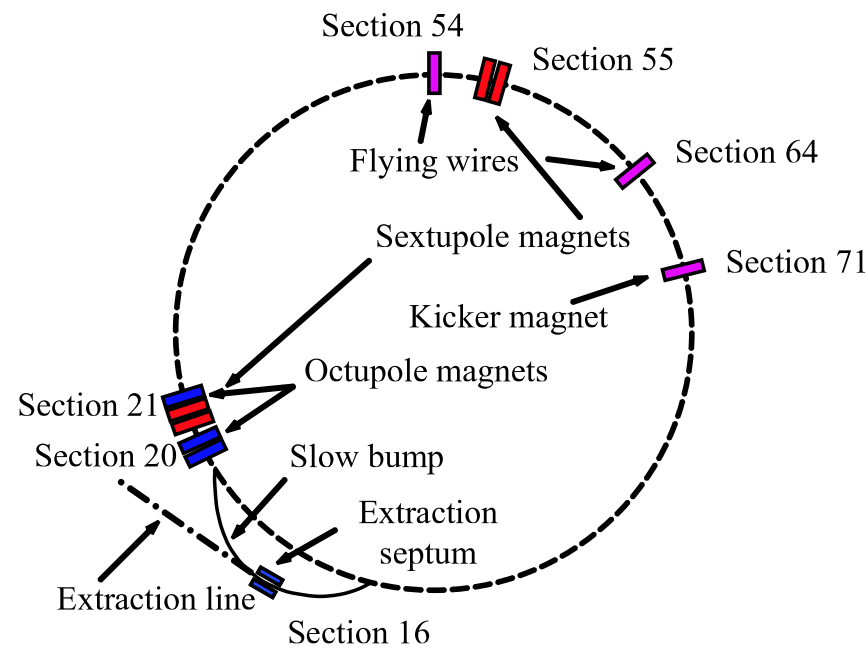

FIG. 1. (Color) Schematic layout of the PS ring with the elements used for the experimental study of the novel multiturn extraction. 
The reconstruction of the horizontal phase space is performed by the standard technique of inducing betatron oscillations and to record them by means of beam position monitors (BPM). In the specific case the extraction kicker, whose modules are located in SS71 and SS79 (see Fig. 1), is used to displace the beam. Furthermore, by changing its strength the whole phase space can be explored. Two pickups normally used for the measurement of the closed orbit are equipped with a fast digitizer so that the beam position can be recorded on a turn-by-turn basis [14].
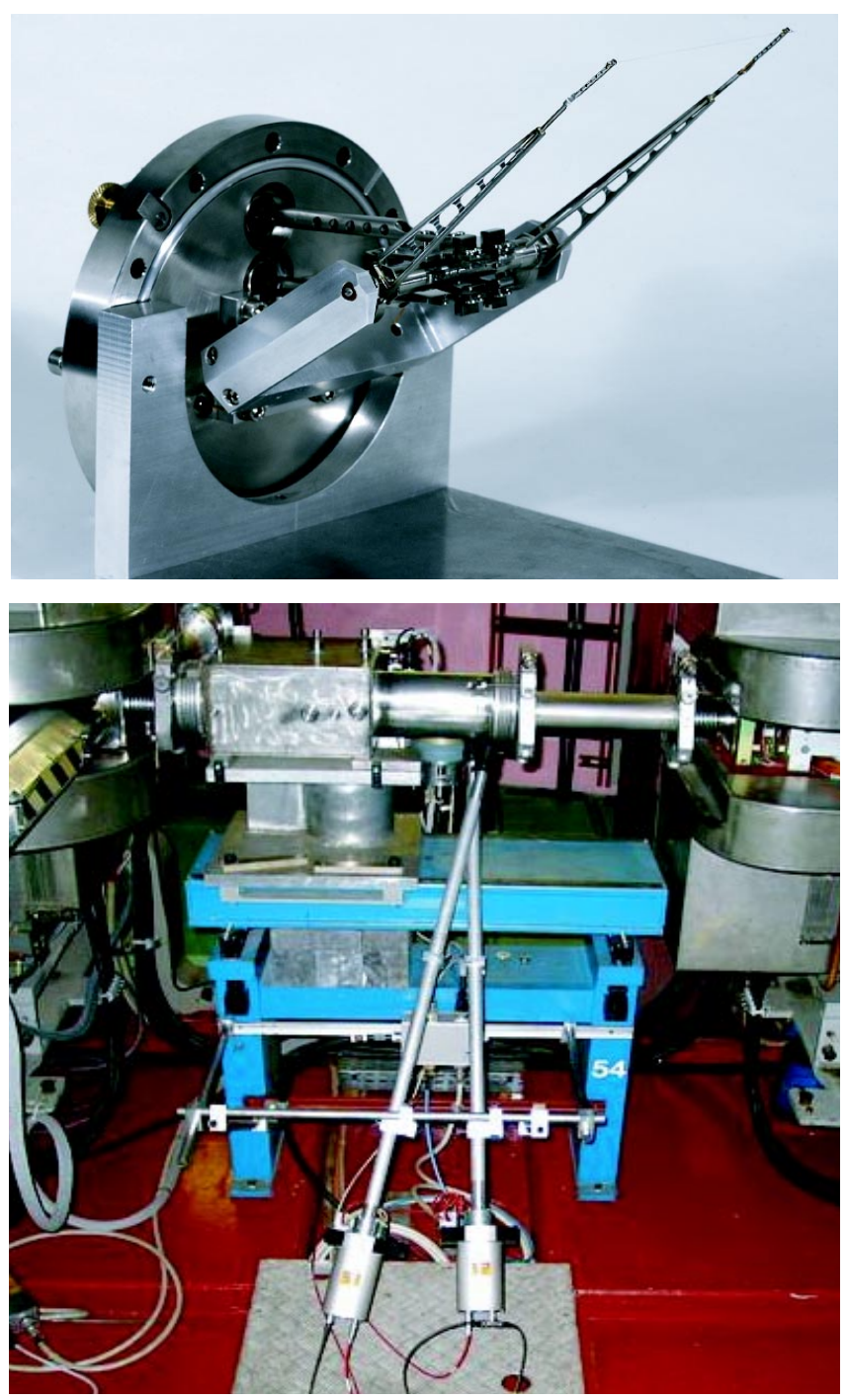

FIG. 2. (Color) Upper panel: Moving parts of the wire scanner. A carbon wire of a diameter of $\approx 50 \mu \mathrm{m}$ is fixed at the end of a moving fork. Lower panel: The moving part rotated by $90^{\circ}$ is mounted inside a vacuum tank to scan the beam in the horizontal direction. A black pill of plastic scintillator located outside the beam vacuum chamber, near its center, is connected to a phototube by a long tube which acts as a light guide. The beam is circulating from left to right. The second scintillator and phototube shown in the photo are not used.
A wire scanner [15] is used to measure the transverse beam profile by detecting the secondary particles generated when a thin carbon wire is moved through the beam. The upper panel of Fig. 2 shows the moving part of the profile monitor. The $\approx 50 \mu \mathrm{m}$ diameter carbon wire is fixed between the tips of the moving fork, which is enclosed inside a vacuum tank once installed in the PS machine (Fig. 2 lower panel). A plastic scintillator connected to a phototube by a light guide and located outside the beam vacuum chamber near its center detects the secondary particles generated by the interaction between the circulating beam and the thin carbon wire. A left-right asymmetry is introduced in the measurement by the fact that a single scintillator located on one side of the vacuum chamber is used for reconstructing the beam profile. While this geometry has a negligible impact on the profile measurement in the case of standard beams, it introduces a nonnegligible asymmetry in the wide and multipeaked profiles observed during the tests.

The recorded beam profile is then used to extract the islands' position, the width of the generated beamlets, as well as the fraction of trapped particles. This is obtained by fitting five (this number is dictated by the resonance order) Gaussian functions $G$ to the raw data and imposing, whenever appropriate, a number of additional constraints. The parametrization used for the fit is given by

$$
\mathcal{G}(x)=\frac{I}{\sqrt{2 \pi} \sigma} e^{-\left[(x-\mu)^{2} /\left(2 \sigma^{2}\right)\right]},
$$

where $I, \mu, \sigma$ represent the integral of the function, the mean value, and the standard deviation, respectively.

An example of the reconstruction technique is shown in Fig. 3, where a typical horizontal beam profile after splitting is reported. The various peaks, corresponding to the particles trapped into the stable islands, are clearly visible. They are fitted with the Gaussian distribution function. The results are superimposed to the measured profile (colored

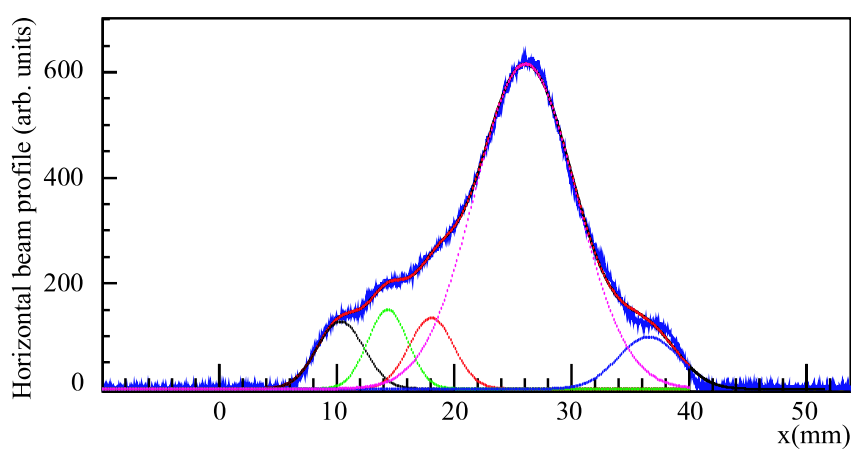

FIG. 3. (Color) Example of measured beam profile after beam splitting. The fitted Gaussian functions are superimposed to the measured profile as well as their sum. The parameters of the beamlets projected onto the center core, such as position, are constrained by using the information from the optical model of the PS machine. 
curves) as well as their sum. Whenever one of the islands is hidden behind the central core, its position is derived from numerical simulations of the phase space topology based on the measured optical model of the PS machine [16] and used as a constraint in the fitting procedure.

A beam current transformer records the beam intensity during the various stages of the experimental tests.

Finally, an optical transition radiation (OTR) monitor [17] is used to record the beam image in the physical $(x, y)$ space during the extraction tests in the transfer line reported in Sec. VII.

\section{PHASE SPACE RECONSTRUCTION}

The measurement of the phase space topology is performed by displacing a low-intensity, single-bunch, pencillike beam, by means of a kicker. The betatron oscillations resulting from this beam perturbation are measured by two pickups, recorded on a turn-by-turn basis and analyzed to detect the presence of stable islands. Under normal conditions, the position signal features a decoherence, due to beam filamentation induced by nonlinear effects and chromaticity, and it tends to zero. However, whenever the beam is displaced inside the acceptance of a stable island, the natural decoherence is almost completely suppressed and this feature can be used as an indicator of the presence of the stable islands. An example of the measurement results is shown in Fig. 4 (see also Ref. [18]). The position signal as a function of turn number is shown in the upper part for the first two-thousand turns. Some damped oscillations are clearly visible in the first part of the time series. These oscillations reveal that the beam is actually rotating around the island's center and this induces some small filamentation. In the subsequent turns, the recorded beam position features a mild decrease. This is probably due to some filamentation induced by the longitudinal motion, which spreads the beam on the separatrix around the stable islands. However, no sign of strong reduction of the signal is visible and an asymptotic value is reached after a few hundred turns.

By combining the signal from two pickups $90^{\circ}$ apart in horizontal phase advance, it is possible to reconstruct the phase space (see Fig. 4 center part), which reveals the position of the four islands. Whenever the first few turns are considered (lower part of Fig. 4), the orbits are found to close up inside each island, as filamentation is not so fast as to influence the signal amplitude at this level. This allows computing an average position, shown as a cross in the figure, representing the position of the island's center. This quantity can be compared with a corresponding analytical estimate based on the knowledge of the PS model [18] (open square in Fig. 4 lower part). An excellent agreement between the two quantities is found for three out of the four islands. A somewhat worse result is obtained for the island in the upper right part of the phase space.
Given the good quality of the measured data, the evaluation of the secondary frequency $\omega_{\mathrm{sec}}$ [19], also called island tune [7], was attempted as well as reconstructing its dependence on the island surface, which is the linear invariant of motion. This gives a detuning curve similarly to what is usually considered for a beam sitting in the center of the phase space. The measured value of $\omega_{\mathrm{sec}}$ is $\omega_{\text {sec }}^{\text {meas }}=0.0375 \pm 0.0008$. It is obtained as an average over
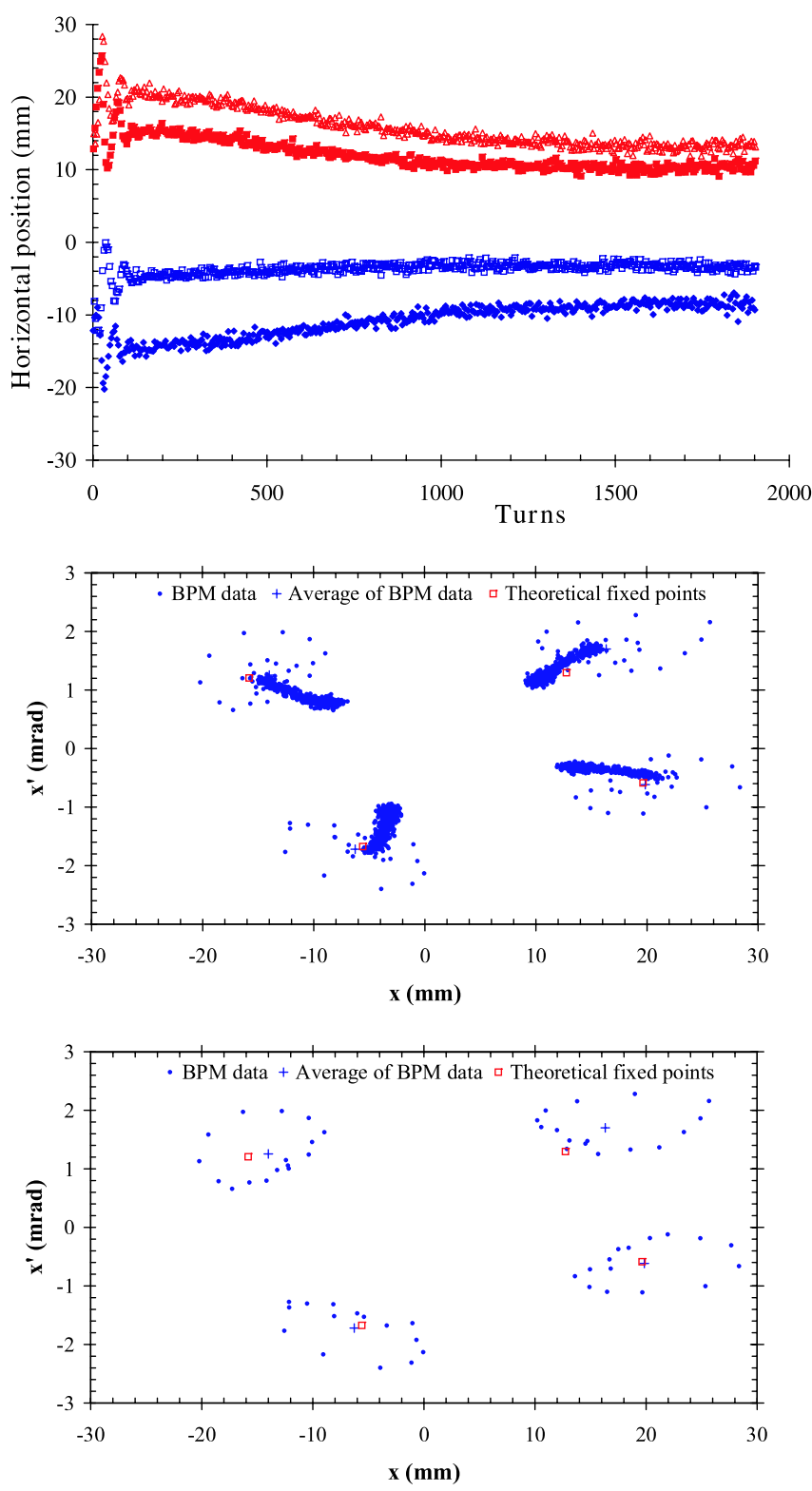

FIG. 4. (Color) Results of the phase space measurements in the presence of stable islands generated by means of sextupoles and octupoles. Upper panel: Beam position as a function of the number of turns. Center panel: Measured horizontal phase space where the position of the fourth-order stable islands is shown. Lower panel: Detail of the first 15 turns. The theoretical position of the fixed points is also shown together with the averaged position over the first 15 recorded turns. 

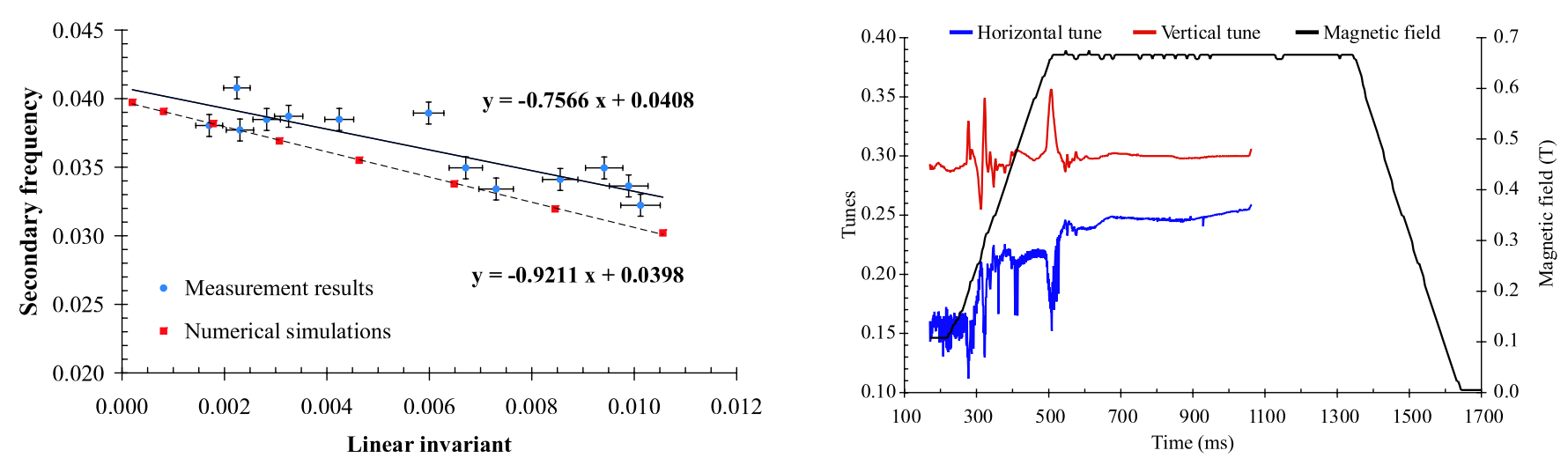

FIG. 5. (Color) Measured secondary frequency $\omega_{\text {sec }}$ as a function of the island surface. The theoretical value $\omega_{\mathrm{sec}}^{\text {th }}$ as a function of the island surface is also reported. The good agreement between the two quantities is clearly visible.

19 different initial amplitudes inside the islands and it is found in good agreement with the predicted value $\omega_{\mathrm{sec}}^{\mathrm{th}}=$ 0.039 [18], based on the measured model of the PS ring [16]. The result of the measurement of the detuning curve inside the stable islands is shown in Fig. 5 (see also Ref. [18]). Even in this case, the agreement between measurement results and analytical estimates is very good.

\section{BEAM SPLITTING TESTS}

The machine is set up in such a way that the islands are created when the fourth-order resonance is crossed starting from a lower tune value. The horizontal tune is set to a value of about 6.245 and it is then increased linearly until the value 6.257 is reached while the vertical tune is kept constant to about 6.3 throughout the splitting process. The evolution of the tune is shown in Figs. 6 and 7. In the first one, the horizontal and vertical fractional tunes are displayed as a function of time together with the magnetic field in the main dipoles from injection to extraction. In the lower panel of Fig. 6, a zoom of the fractional tune evolution in the neighborhood of the resonance crossing is shown. It is important to stress that not much effort was devoted to the optimization of the low-energy tunes, since it has no impact on the efficiency of the trapping process. This explains the rather erratic behavior of the measured curves. On the other hand, the choice of the tune during the magnetic flattop and, in particular, during the beam splitting was optimized with care. Interestingly enough, when the resonance is crossed the vertical tune features also a linear variation during a short lapse of time. This might be induced by the nonlinear coupling enhanced during the resonance-crossing stage. The tune evolution in the tune space is reported in Fig. 7, together with the resonance lines from order 4 to 9 . Even though the crossing of the fourth-order resonance occurs at the intersection of the lines corresponding to the seventh-order resonances $(2, \pm 5)$, this working point is the one giving the minimum amount of beam losses.

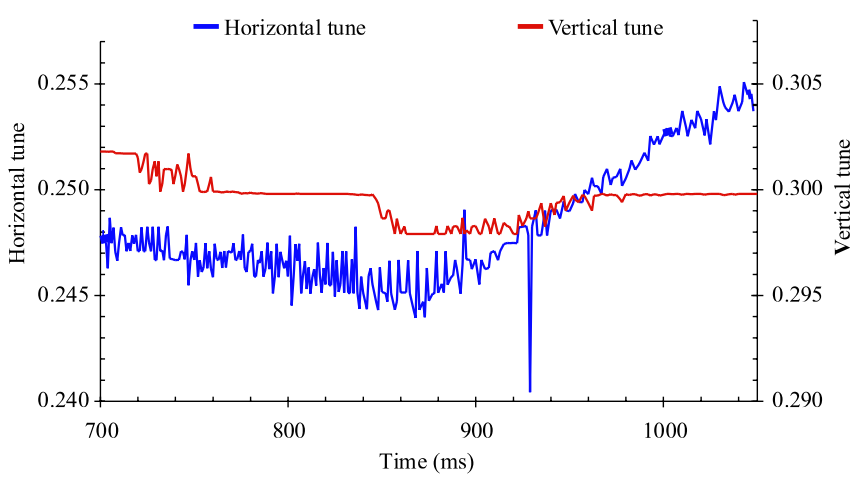

FIG. 6. (Color) Upper panel: Measured horizontal and vertical fractional tune as a function of time together with the PS main dipoles' magnetic cycle. Lower panel: Horizontal and vertical tune evolution vs time in the interval where the fourth order resonance crossing occurs.

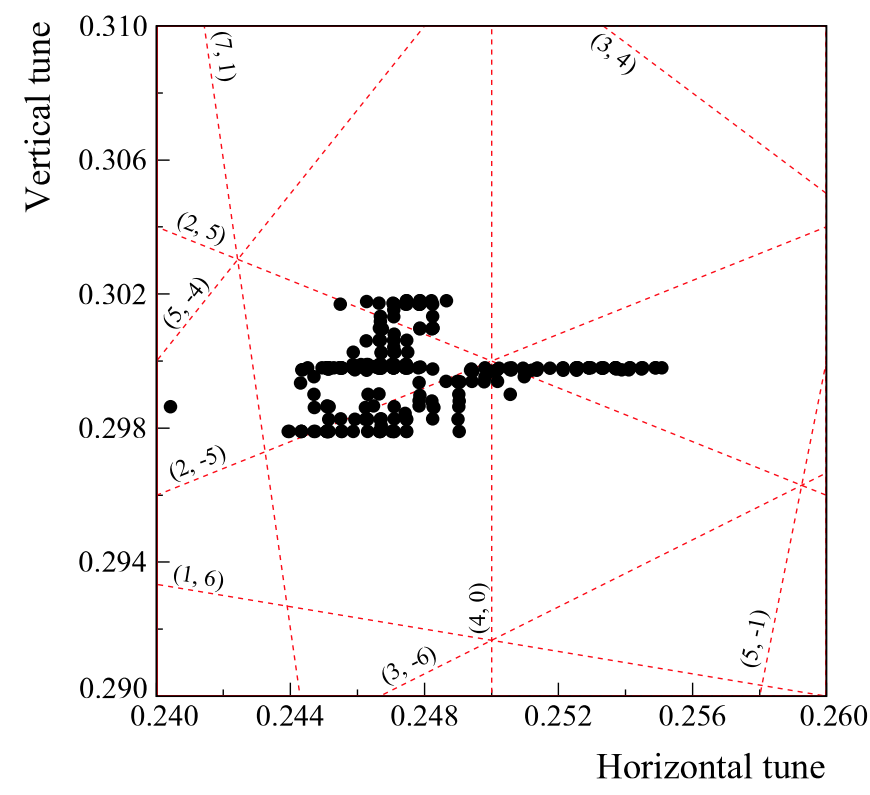

FIG. 7. (Color) Tune working diagram. The dots represent the fractional tune evolution in time measured during the beam trapping and splitting process. Only the points shown in the lower panel of Fig. 6 are reported here. 
The tests with the low-intensity beam are performed by keeping the strength of the sextupoles and octupole constant during the resonance-crossing stage. The test results are plotted in Fig. 8, where the horizontal beam profile for a single-bunch, low-intensity beam is shown for three values of the horizontal tune. The initial Gaussian profile (left part) is deformed when crossing the resonance (central part). The tails represent the beam trapped inside the stable islands: by varying the tune the beamlets can be completely separated from the beam core (right part) so that no particle populates the region between the outermost beamlet and the beam core. Even more important, during the beam manipulation no detectable particle loss is observed. These results indicate that the principle of trapping inside stable islands is indeed working well. It is important to stress that the wire scanner does not allow recording more than one beam profile during the same cycle. Therefore, the three profiles represent views over three magnetic cycles. Thanks to the good reproducibility of the PS machine and of the proposed beam manipulation, such an approach produces reliable and stable results.

When the tests with the high-intensity beam started, the same configuration of the PS machine found during capture tests with the low-intensity one was initially used. Losses at the level of $10 \%-15 \%$ of the total beam intensity were observed during the resonance-crossing phase. The optimization of the vertical tune allowed decreasing the losses down to less than $5 \%$, while the threshold of $1 \%$ was broken by reducing the octupole strength during the process of resonance-crossing. The change of the octupole strength induces a displacement towards higher amplitudes of the islands. Therefore, it is possible to achieve the same final beamlets' separation while applying a reduced tune variation.

A careful compensation of the beam-loading effects in the radio-frequency cavities via the feedback loops led to a reduction of the beam losses well below the threshold of detection of the beam current transformer. This can be seen

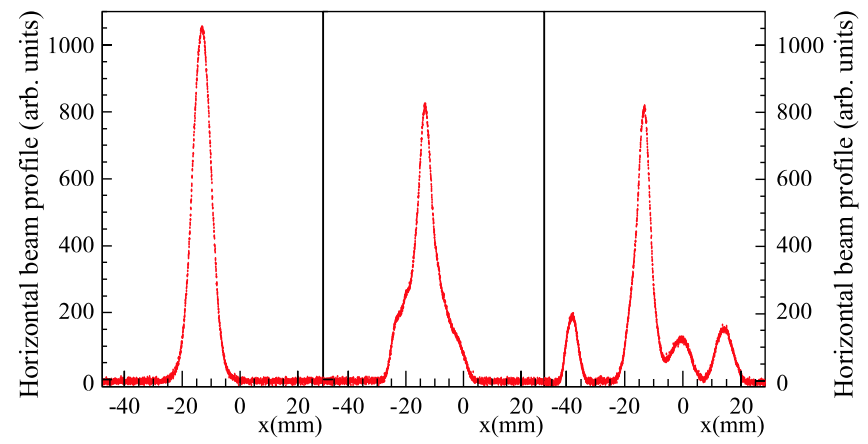

FIG. 8. (Color) Evolution of the horizontal beam distribution during the resonance crossing. The initial beam distribution is shown on the left; in the center the resonance is just crossed and the heavy tail are visible; on the right the final stage is shown: well-separated beamlets are clearly visible. in Fig. 9 (upper panel) where the experimental conditions for the high-intensity tests are reported as a function of time. The magnetic cycle is shown together with the beam intensity, the strength of the two families of tuning quadrupoles and the powering of the sextupoles and the single octupole used to generate the stable islands. The time interval when the resonance is crossed is highlighted by two vertical lines. The intensity does not feature any variation with time, apart from some fluctuations due to measurement errors. Under these conditions the fraction of particles trapped in each of the four beamlets, as obtained from the fitted Gaussian, was about $14 \%$ of the initial beam distribution.

Having achieved the first goal of showing the feasibility of a loss-free beam splitting, the second goal consisted in increasing the fraction of particles trapped inside the islands so to reach the target of $20 \%$, i.e., equal beam sharing in the five beamlets. This series of tests was carried out by
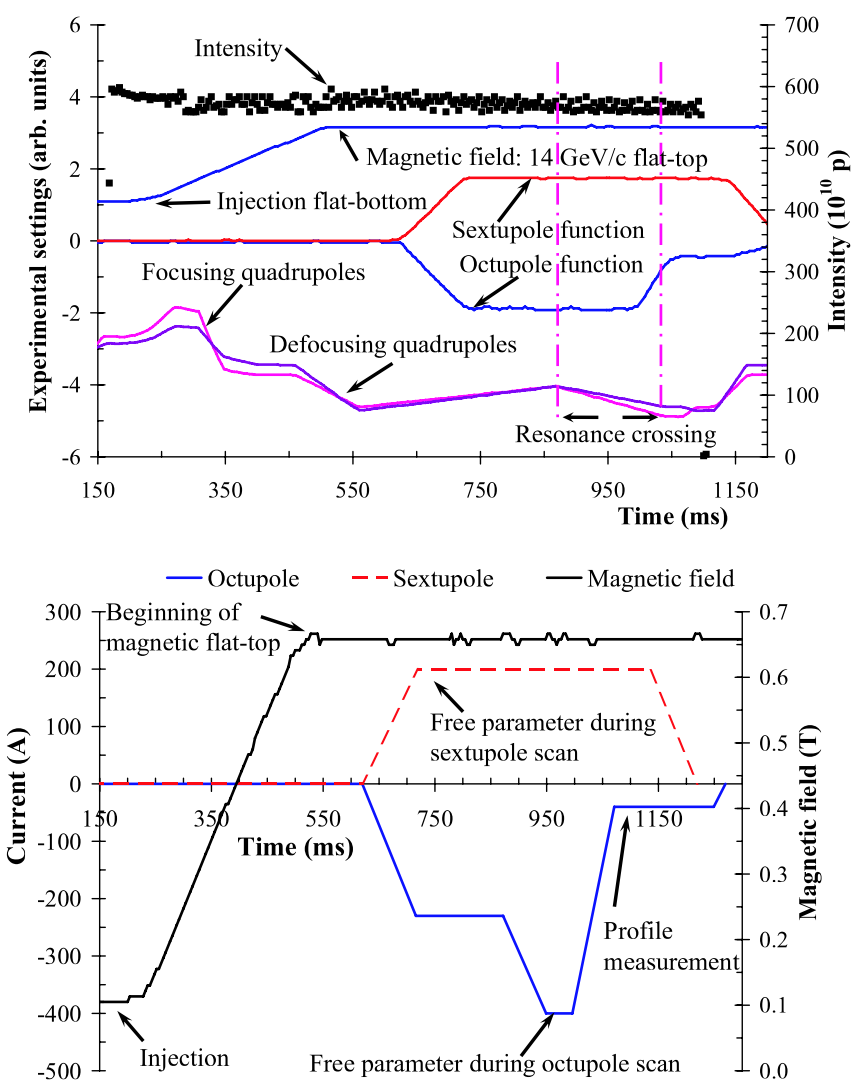

FIG. 9. (Color) Upper panel: Experimental conditions for the high-intensity trapping tests, namely, magnetic field of the main dipoles, strength of the focusing and defocusing quadrupole families used to cross the fourth-order resonance, strength of the sextupoles and octupole, and beam intensity as a function of time. Lower panel: Experimental conditions for the highintensity trapping tests aimed at increasing the fraction of trapped particles, namely, magnetic field of the main bending magnets and strength of the sextupoles and octupole as a function of time. 

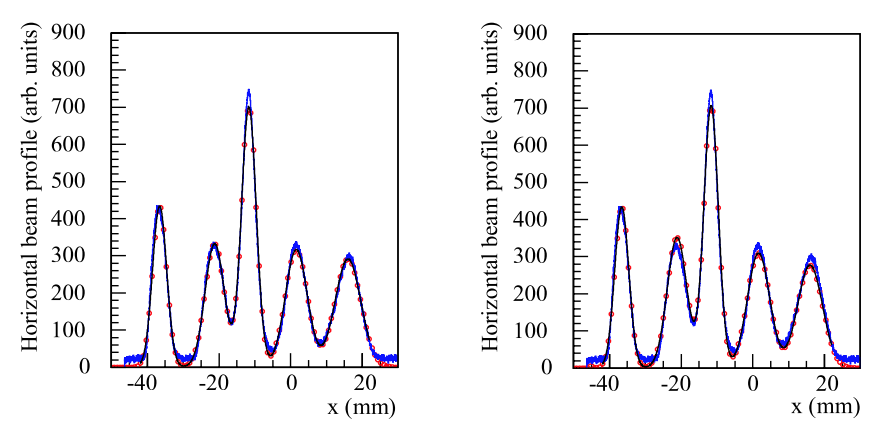

FIG. 10. (Color) Horizontal beam profile at the end of the trapping and separation process with fit based on five independent Gaussian functions (left) or three Gaussian functions with the same amplitude plus two independent ones (right). The red dots represent the fit results.

scanning over the strength of the nonlinear elements. Only a rough study for the variation of the maximum current for the sextupoles was performed. Indeed, the sextupoles have an impact on the machine chromaticity. Thus, after each change of the sextupoles' strength, a correction of the linear chromaticity should have been performed. Because of the layout of the PS ring, the chromaticity correction is a long and tedious process. Therefore, it was decided to limit the detailed study of the optimization of the beam capture to the analysis of the impact of the octupole, which has no influence on the linear chromaticity and thus does not require its continuous correction.

By scanning over the octupole's strength (see Fig. 9 lower panel), it was possible to increase the fraction of particles trapped in the beamlets. The best result achieved is shown in Fig. 10, where an average fraction of $18 \%$ of the total initial beam intensity is trapped in the beamlets. The same figure shows also an example of the very good quality of the fit results. The same set of raw data is fitted by means of five independent Gaussian functions (left), or by five functions with an additional constraint. In the case under discussion, the three rightmost beamlets should contain the same fraction of trapped particles (right). This constraint is a consequence of the geometry of the experimental setup that induces left/right asymmetry in the mea-

TABLE II. Summary of the values of the fit parameters obtained using five independent Gaussian functions applied to the best result obtained in terms of fraction of captured particles. The Gaussian functions are numbered from left to right according to Fig. 10.

\begin{tabular}{cccc}
\hline \hline $\begin{array}{c}\text { Variable physical } \\
\text { parameter }\end{array}$ & $\begin{array}{c}I \\
\text { (arbitrary units) }\end{array}$ & $\begin{array}{c}\mu \\
(\mathrm{mm})\end{array}$ & $\begin{array}{c}\sigma \\
(\mathrm{mm})\end{array}$ \\
\hline$G_{1}$ & 2152.1 & -36.5 & 1.98 \\
$\mathcal{G}_{2}$ & 2240.5 & -21.2 & 2.69 \\
$\mathcal{G}_{3}$ & 3650.8 & -11.6 & 2.08 \\
$\mathcal{G}_{4}$ & 2447.0 & 1.6 & 3.07 \\
$\mathcal{G}_{5}$ & 2638.3 & 16.0 & 3.61 \\
\hline \hline
\end{tabular}

TABLE III. Summary of the values of the fit parameters obtained using five Gaussian functions with fitting constraints applied to the best result obtained in terms of fraction of captured particles. The Gaussian functions are numbered from left to right according to Fig. 10.

\begin{tabular}{cccc}
\hline \hline $\begin{array}{c}\text { Variable physical } \\
\text { parameter }\end{array}$ & $\begin{array}{c}I \\
\text { (arbitrary units) }\end{array}$ & $\begin{array}{c}\mu \\
(\mathrm{mm})\end{array}$ & $\begin{array}{c}\sigma \\
(\mathrm{mm})\end{array}$ \\
\hline$G_{1}$ & 2152.1 & -36.5 & 1.98 \\
$G_{2}$ & 2428.4 & -21.2 & 2.75 \\
$G_{3}$ & 3618.4 & -11.6 & 2.05 \\
$G_{4}$ & 2428.4 & 1.6 & 3.13 \\
$G_{5}$ & 2428.4 & 16.0 & 3.49 \\
\hline \hline
\end{tabular}

sured beam profiles. Some losses, of the order of $2 \%-3 \%$ of the total beam intensity, were observed when crossing the resonance in correlation with an unstable behavior in the longitudinal plane. Hence, those losses might be induced by problems with the longitudinal dynamics, rather than being an intrinsic limitation due to the nonlinear effects in the transverse planes. A summary of the values of the fit parameter is reported in Tables II and III.

\section{DETAILED MEASUREMENTS}

\section{A. Reversibility tests of the trapping process}

To gain insight on the details of the process in view of improving the control of the final beamlets' properties and to qualify the robustness of the method against perturbations, extensive tests were performed using the lowintensity beam. The issue of whether the proposed beam manipulation is reversible is an aspect studied in detail. This point has certainly a high relevance from the theoretical point of view, but it is also crucial for the future implementation, e.g., for defining the minimum time required to cross the resonance so to leave the final beam parameters unaffected. In fact, on one side the particles' trapping has to be slow (adiabatic) to limit the disturbance due to the nonlinear effects. On the other side the time available to split the beam is limited, mainly by the duration of the PS magnetic cycle. During the finite lapse of time corresponding to the magnetic flattop many other beam manipulations need to take place and not all of them can be performed in parallel with each other. A rough estimate of the time available for beam splitting is about $50-90 \mathrm{~ms}$.

The experimental procedure consisted in crossing the resonance twice, in opposite directions, and in measuring the horizontal beam profile before the first crossing and after the horizontal tune is brought back to its initial value. In addition, a control measurement of the beam profile after the first crossing was performed to assess the fraction of particles trapped. Such a control measurement was made when the tune was kept constant after the first resonance crossing. Two sets of measurements were taken corre- 
sponding to two durations of the stage with constant tune, respectively $20 \mathrm{~ms}$ and $180 \mathrm{~ms}$. Typical results are shown in Fig. 11, where the horizontal beam profiles before, after the double crossing, as well as the control profile are displayed for a fast resonance crossing (5 ms, left panel) and a slow crossing ( $90 \mathrm{~ms}$, right panel). In both cases the duration of the constant tune stage is $180 \mathrm{~ms}$. The difference between the two cases is apparent. Fast crossing implies that only a small fraction of particles is trapped inside the islands. Nevertheless, the difference between the beam profiles before and after coming back to the initial tune is hardly visible. On the other hand, slow crossing implies that a larger fraction of particles is trapped, but under these conditions the final beam profile features heavy tails. The distribution looks like the superposition of two Gaussian functions with the same average value, but different sigma values. In this respect, the experimental data seem to indicate that the process is not reversible. From the observation that the results hardly depend on the duration of the stage with constant tune, it is possible to rule out phenomena such as periodic modulation of the linear tune as sources of the nonreversibility. A better candidate could be the nonlinear coupling between the horizontal and vertical degrees of freedom. In fact, even though great care was devoted to minimizing the disturbances in the vertical plane, e.g., by locating the sextupoles and octupole where the vertical beam size is small, such a coupling is never zero. Hence, despite the fact that the horizontal tune is brought back to its initial value the global system is not in its original state. Theoretical analysis of the details of the trapping process is in progress to clarify this aspect.
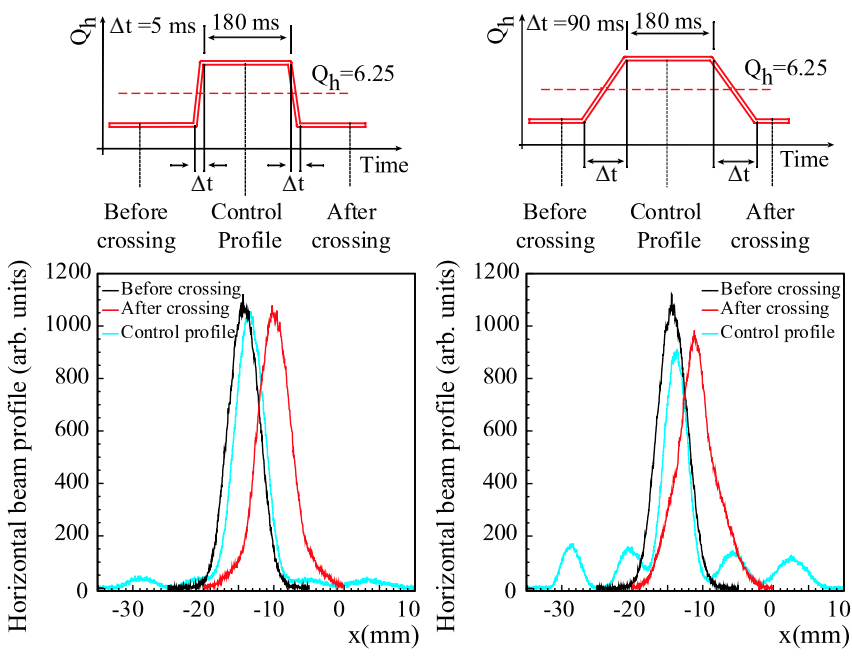

FIG. 11. (Color) Left panel: Horizontal beam profiles for a fast (5 ms) crossing of the resonance. Right panel: Horizontal beam profiles for a slow $(90 \mathrm{~ms})$ crossing of the resonance. The difference in fraction of trapped particles for the two crossing speeds is clearly visible in the control profiles, as well as the presence of thick tails in the case of slow crossing in the final profile.
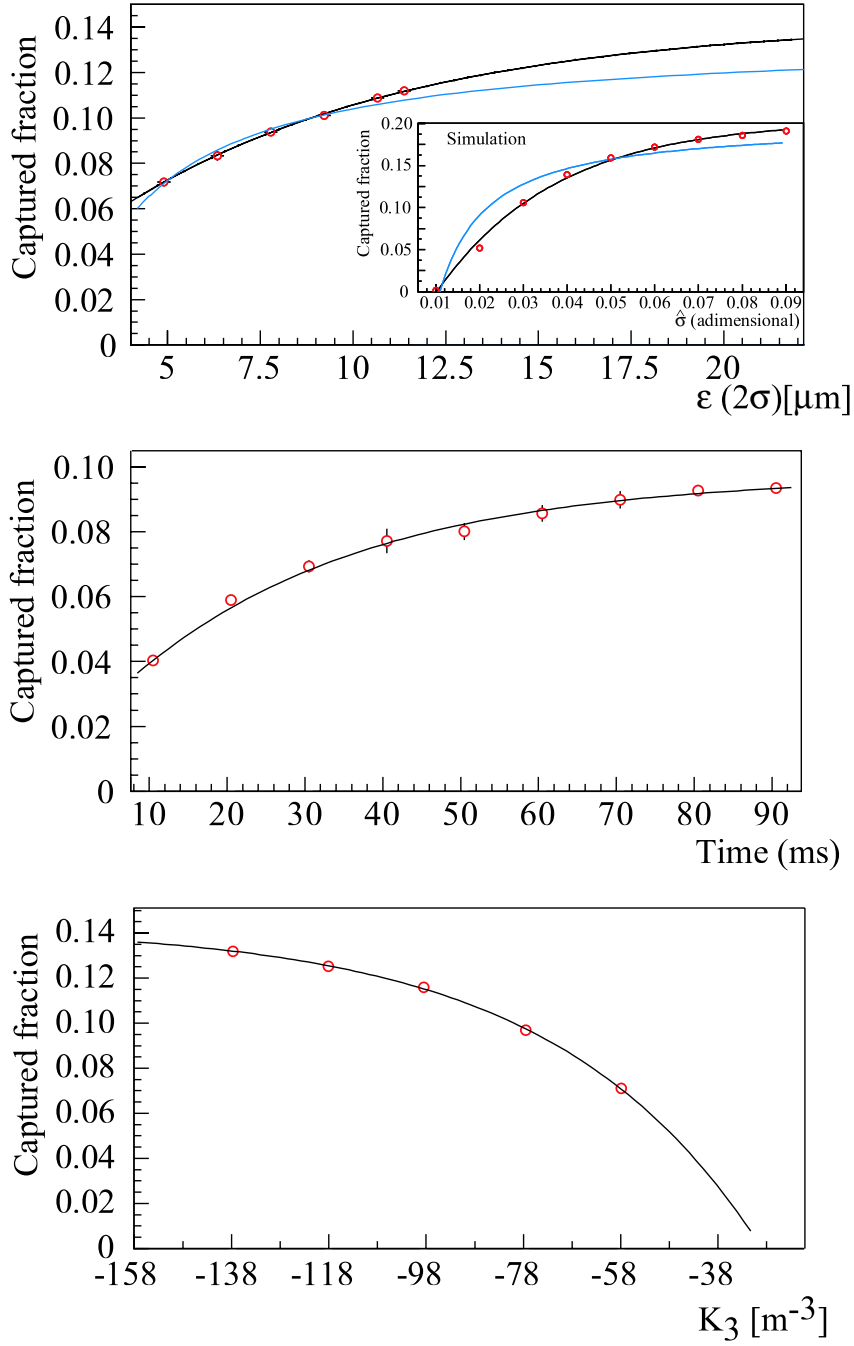

FIG. 12. (Color) Fraction of initial beam intensity trapped in the four beamlets as a function of the emittance of the incoming beam (upper panel) for both measured and simulated data (inset, see Refs. [1,2] for the definition of $\hat{\sigma}$ ), the time of the resonancecrossing process (center panel), and the octupole strength $K_{3}$ (lower panel). The experimental data are fitted with a function $y=a+b e^{-c x}$ (black line). The fitting function $y=a+b / x$ is also used for the upper plots (light line). The error bars correspond to an rms value computed over five sets of experimental data.

From a practical point of view, one can state that, using a linear tune variation, the fraction of particles trapped in the beamlets does not change substantially provided the crossing time is longer than $50-70 \mathrm{~ms}$ (see also Fig. 12, center panel).

\section{B. Trapping process optimization}

A series of tests were performed to study how to share equally emittance and intensity among the five beamlets. Measuring the emittance of each beamlet is conceptually a nontrivial issue and, in any case, it is beyond the capabil- 
TABLE IV. Fit parameters of the curve $y=a+b e^{-c x}$ applied to the three series of measured data plotted in Fig. 12. The value of the fit parameters for the function $y=a+b / x$ are reported in parenthesis.

\begin{tabular}{llll}
\hline \hline Variable physical parameter & \multicolumn{1}{c}{$a$} & \multicolumn{1}{c}{$b$} & \multicolumn{1}{c}{$c$} \\
\hline Emittance incoming beam $(\mu \mathrm{m})$ & $+0.143(+0.135)$ & $-0.133(-0.315)$ & $+0.128 \mu \mathrm{m}^{-1}$ \\
Time resonance crossing $(\mathrm{s})$ & $+0.097(+0.098)$ & $-0.081(-0.61)$ & $+0.034 \mathrm{~s}^{-1}$ \\
Octupole strength $\left(\mathrm{m}^{-3}\right)$ & $+0.143(+0.179)$ & $-0.292(+5.969)$ & $-0.025 \mathrm{~m}^{3}$ \\
\hline \hline
\end{tabular}

ities of the PS instrumentation. Hence, the study focused on the issue of intensity sharing. As already mentioned, the ultimate goal consists in trapping $20 \%$ of the initial beam intensity in each beamlet. The dependence of the fraction of particles trapped inside the beamlets on three selected parameters was probed by performing detailed scanning studies. The parameters considered for these studies were: (i) the emittance of the incoming beam; (ii) the duration of the resonance-crossing stage (see previous section); (iii) the octupole strength. The results are shown in Fig. 12 (open markers) together with some fitting curves. The error bars represent the one-sigma level of the error obtained by taking the average over a set of five experimental data.

The case of the emittance of the incoming beam is displayed in the upper panel. By appropriate manipulations performed at the level of the PS booster, it is possible to act on the value of the horizontal emittance, while leaving both the vertical one and the total beam intensity almost constant. In the inset, the results from numerical simulations performed on a simple model are reported for comparison. The simulation data are used to derive a phenomenological fitting curve to be then applied to the measured data. To this aim, the function $y=a+b e^{-c x}$ was used (black curve in Fig. 12), together with an alternative function of the form $y=a+b / x$ (light curve in the upper panel of Fig. 12). This choice is made to have a fitting function featuring an horizontal asymptote, thus allowing also to check the robustness of the estimate of the asymptotic value $a$ as a function of the choice of the fitting function. Clearly, the exponential curve gives the best fitting results and it was used also for the other experimental results.

In the case of the dependence of the fraction of the trapped particles on the time spent in crossing the resonance (see Fig. 12 center panel), the experimental procedure was very similar to the one used to study the reversibility of the process, but with a single resonance crossing performed in one direction, only.

Finally, in the case of the dependence of the fraction of particles trapped as a function of the octupole's strength, the results are reported in the lower panel of Fig. 12. As shown in Fig. 9 (lower panel), the value of the octupole's strength corresponding to the negative peak is scanned, while keeping the other details of the function unchanged.

In all three cases, the experimental data feature a very smooth dependence on the physical parameter under study. Furthermore, the exponential function proves to fit the measured data remarkably well. The results of the two fits, with exponential or hyperbolic dependence on the physical parameter, are summarized in Table IV. It is worth stressing that the value of the fit parameter $a$ turns out to be to a large extent independent from the functional form of the fitting function. Indeed, the difference between the value of $a$ for the exponential or hyperbolic function is of the order of a few percent, the maximum difference being $25 \%$.

As a final confirmation of the strong impact of the octupole's strength on the capture process, a series of horizontal beam profiles measured at the end of the capture process are shown in Fig. 13 as a function of the normalized integrated gradient $K_{3}=L /(B \rho) d^{3} B_{y} / d x^{3}$, where $L$ stands for the octupole length, $B \rho$ the magnetic rigidity, and $B_{y}$ the vertical component of the magnetic field in the octupole.

These profiles correspond to the data series presented in Fig. 12 (lower panel) and visualize the mechanism by

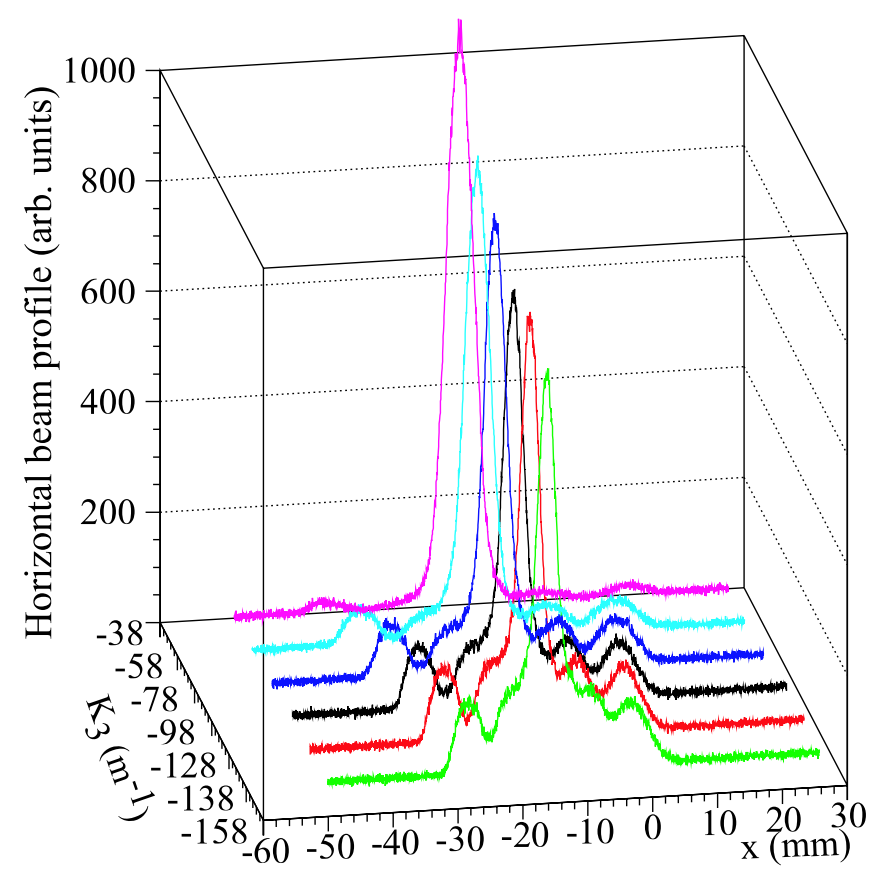

FIG. 13. (Color) Horizontal beam profiles as a function of the octupole strength $K_{3}$ at the end of the capture process. The change in the fraction of trapped particles, as well as the change in beamlets' position, is clearly visible. 
which the octupole strength affects the trapping process. In fact, it acts on the size of the islands within which the particles are trapped, thus improving the capture and, at the same time, it changes the islands' position by varying the detuning with amplitude.

Similar tests were carried out also with the highintensity beam and the results do not differ qualitatively from those presented here. This indicates, once more, that space charge effects have a very limited impact on the process under study at $14 \mathrm{GeV} / c$, in spite of the fact that the beam intensities differ by more than an order of magnitude for the various flavors of the proton beam used for the tests.

\section{FAST EXTRACTION TESTS}

Using the hardware currently installed in the CERN PS, it is not possible to extract the beamlets over many turns. Therefore testing the complete process of multiturn extraction, from beam splitting to extraction, is not feasible. Nevertheless, it is indeed possible to extract the entire beam in one machine turn by means of the hardware normally used to perform a fast extraction. In this case, after having split the beam, the horizontal tune is slightly reduced in order to decrease the islands' separation. This is imposed by the need of matching the overall beam size with the extraction septum aperture and that of the downstream transfer line. A kick is imparted to the whole structure, which starts oscillating around the closed orbit and then it jumps beyond the extraction septum and is deflected towards the PS-SPS transfer line.

An OTR monitor [17] is used to record the beam image in the physical space as presented in the right part of Fig. 14. Side lobes are clearly visible, which represent two separated beamlets to the left and to the right of the beam core, respectively. The two remaining beamlets (below and above the central core, see left part of Fig. 14) are then projected onto the central core, thus increasing its intensity as seen on the OTR screen image. This result

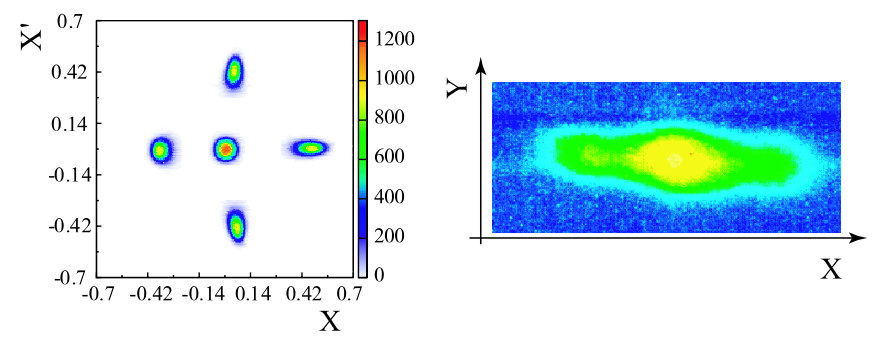

FIG. 14. (Color) Right panel: Transverse phase space in normalized coordinates showing the beamlets at the end of the capture process according to numerical simulations. Left panel: Twodimensional beam distribution in physical space of the split beam in the transfer line downstream of the PS extraction point. The image was obtained by means of an OTR detector. The lateral lobes are generated by the projection of the partially separated beamlets (see left panel). proves that the whole split beam can be transported in the transfer line while preserving its peculiar structure.

\section{CONCLUSIONS}

An intense experimental campaign at the CERN PS demonstrated the feasibility of a loss-free adiabatic splitting of a charged particle beam using stable islands of transverse phase space. The stable islands are created by a set of special magnets, sextupoles, and octupoles, and the beam trapping is obtained by crossing the fourth-order resonance. With such a scheme five beamlets are created, with four of them sharing the same fraction of initial beam fraction. The amount of beam in the beamlets depends both on the initial emittance and on the octupole gradient. The beamlets can be completely separated in phase space by properly adjusting the value of the machine fractional tune or the octupole's gradient at the end of the resonancecrossing process. Such a separation could be used to extract one by one the different beamlets with virtually no losses.

In this paper not only the general results from the tests of beam splitting were reported, but also a number of detailed measurements aimed at improving the general understanding of the splitting process in view of a further optimization of the whole process.

Following the positive results of the experimental campaign, the analysis of a possible implementation of the proposed scheme in the PS ring was performed. Currently, the design stage is finished and the final layout is ready for approval. A detailed description of the proposed PS extraction scheme can be found in [20]. According to this proposal the new multiturn extraction should be implemented and completed during the next two years with the goal of starting its commissioning with beam in 2008, when it will deliver beam to the CNGS in parallel with the present scheme. Upon completion of this phase, the CT scheme will be stopped and the hardware decommissioned so that from 2009 onwards the new multiturn extraction will be the only scheme in operation for providing beam to CNGS.

\section{ACKNOWLEDGMENTS}

We are particularly indebted to R. Cappi, the originator of the proposal to use stable resonances to split the beam, and to J.-P. Riunaud who had a key role in supporting and encouraging the research. We warmly acknowledge the contribution of M. Benedikt and M. Chanel for preparing the PS Booster beams and S. Hancock for the support with the longitudinal beam dynamics in the PS.

[1] R. Cappi and M. Giovannozzi, Phys. Rev. Lett. 88, 104801 (2002).

[2] R. Cappi and M. Giovannozzi, Phys. Rev. ST Accel. Beams 7, 024001 (2004). 
[3] M. Giovannozzi and J. Morel, in Proceedings of the 2005 Particle Accelerator Conference, edited by C. Horak (IEEE Computer Society Press, Piscataway, 2006), p. 1377.

[4] L. Bongini, A. Bazzani, G. Turchetti, and I. Hofmann, Phys. Rev. ST Accel. Beams 4, 114201 (2001).

[5] R. Garoby, CERN-PS-2001-004-RF, 2001.

[6] A. Chao, D. Johnson, S. Peggs, J. Peterson, C. Saltmarsh, L. Schachinger, R. Meller, R. Siemann, R. Talman, P. Morton, D. Edwards, D. Finley, R. Gerig, N. Gelfand, M. Harrison, R. Johnson, N. Merminga, and M. Syphers, Phys. Rev. Lett. 61, 2752 (1988).

[7] S. Y. Lee, M. Ball, B. Brabson, D. D. Caussyn, J. Collins, S. Curtis, V. Derenchuck, D. DuPlantis, G. East, M. Ellison, T. Ellison, D. Friesel, B. Hamilton, W. P. Jones, W. Lamble, D. Li, M. G. Minty, P. Schwandt, T. Sloan, G. $\mathrm{Xu}$, A. W. Chao, S. Tepikian, and K. Y. Ng, Phys. Rev. Lett. 67, 3768 (1991).

[8] R. Cappi and M. Giovannozzi, in Proceedings of Eighth Particle European Accelerator Conference, edited by J. Poole and C. Petit-Jean-Genaz (Institute of Physics, London, 2002), p. 1250.

[9] R. Cappi, M. Giovannozzi, M. Martini, E. Métral, G. Métral, R. Steerenberg, and A.-S. Müller, in Proceedings of the 2003 Particle Accelerator Conference, edited by J. Chew, P. Lucas, and S. Webber (IEEE Computer Society Press, Piscataway, 2003), p. 388.

[10] M. Giovannozzi, R. Cappi, S. Gilardoni, M. Martini, E. Métral, A. Sakumi, R. Steerenberg, and A.-S. Müller, in Proceedings of the Ninth European Particle Accelerator Conference, edited by J. Poole and C. Petit-Jean-Genaz (Institute of Physics, London, 2004), p. 173.

[11] G. Acquistapace, J.-L. Baldy, A. E. Ball, P. Bonnal, M. Buhler-Broglin, F. Carminati, E. Cennini, A. Ereditato, V. P. Falaleev, P. Faugeras, A. Ferrari, G. Fortuna, L. Foà, R. Genand, A. L. Grant, L. Henny, A. Hilaire, K. Hübner, J. Inigo-Golfin, K. H. Kissler, L. A. López-Hernandez,
J.-M. Maugain, M. Mayoud, P. Migliozzi, D. Missiaen, V. Palladino, I. M. Papadopoulos, F. Pietropaolo, S. Péraire, S. Rangod, J.-P. Revol, J. Roche, P. R. Sala, C. Sanelli, G. R. Stevenson, B. Tomat, E. Tsesmelis, R. Valbuena, H.H. Vincke, E. Weisse, and M. Wilhelmsson, CERN 98-02, 1998, edited by K. Elsener.

[12] K. Cornelis, J.-P. Delahaye, R. Garoby, H. Haseroth, K. Hübner, T. Linnecar, S. Myers, K. Schindl, and C. Wyss, CERN PS-2001-041 (AE), 2001, edited by R. Cappi.

[13] O.E. Berrig, J. Borburgh, J.-P. Burnet, R. Cappi, W. Kalbreier, M. Martini, A.-S. Müller, E. Métral, K.-D. Metzmacher, J.-P. Riunaud, A. Sakumi, P. Scaramuzzi, L. Sermeus, R. Steerenberg, and T. Zickler, CERN AB2004-003, 2004, edited by M. Giovannozzi.

[14] M.E. Angoletta and A.S. Müller, in Proceedings of Eighth Particle European Accelerator Conference, Ref. [8], p. 1948.

[15] C. Steinbach and M. Van Rooij, IEEE Trans. Nucl. Sci. 32, 1920 (1985).

[16] R. Cappi, M. Giovannozzi, M. Martini, E. Métral, G. Métral, R. Steerenberg, and A.-S. Müller, in Proceedings of the 2003 Particle Accelerator Conference, Ref. [9], p. 2913.

[17] C. Bovet and R. Jung, LHC Project Report 3, 1996.

[18] M. Giovannozzi and P. Scaramuzzi, in Proceedings of the Ninth European Particle Accelerator Conference, Ref. [10], p. 1861.

[19] A Bazzani, G. Servizi, E. Todesco, and G. Turchetti, CERN Yellow Report, 94-02, 1994.

[20] M.J. Barnes, O.E. Berrig, A. Beuret, J. Borburgh, P. Bourquin, R. Brown, J.P. Burnet, F. Caspers, J. M. Cravero, T. Dobers, T. Fowler, S. Gilardoni, M. Hourican, W. Kalbreier, T. Kroyer, F. di Maio, M. Martini, V. Mertens, E. Métral, K. D. Metzmacher, C. Rossi, J. P. Royer, L. Sermeus, R. Steerenberg, G. Villiger, T. Zickler, CERN Report, 2006-011, 2006, edited by M. Giovannozzi. 